\title{
CAWriter: A CSCW/CSCL Tool to Support Research Students' Academic Writing
}

\author{
Jake Rowan Byrne \\ Brendan Tangney \\ Centre for Research in IT in Education, School of Education and School of \\ Computer Science \& Statistics, Trinity College, Dublin, Ireland \\ byrnejr@tcd.ie \\ tangney@tcd.ie
}

\begin{abstract}
Within the larger framework of a research project aiming to develop CSCW/CSCL tools, to scaffold a cognitive apprenticeship model as applied to doctoral education, academic writing has been identified as a key process to be supported. By following a participatory design approach, and by building upon previous writing tools, a prototype is being developed to assist Computer Science research students in the academic writing process. Key features of the tool are that it supports non-linear document creation and multiple representations of notes and document content. Initial feedback from use of the tool and planned future work to integrate collaboration to support cognitive apprenticeship are reported.
\end{abstract}

Participatory Action Research, Participatory Design, Writing Tools, Cognitive Apprenticeship, Research Methods, Computer Supported Collaborative Learning

\section{INTRODUCTION}

This paper describes CAWriter a tool which combines the advantages of existing writing tools and places them in an online environment. It is argued that the online approach allows future development to enter a more flexible and mobile space than the previous desktop equivalents, enabling the user to access their resource in a number of contexts, therefore supporting a number of cognitive apprenticeship practices.

An overview of related work is followed by a discussion of the participatory design methodology used and the design of the prototype. Initial testing is reported upon before discussion.

\section{RELATED WORK Cognitive Apprenticeship}

Cognitive apprenticeship builds on the traditional craft apprenticeship model where the learner acquires, develops and uses skills obtained from the practices of experts through observation and collaboration in an authentic social context [4]. In this view, and that of situated learning [9], the locus of learning expands beyond the more common interpretation of only occurring within the individuals' mind to that of a more socially distributed processes, in what Lave and Wegner call "legitimate peripheral participation" in a "community of practice". Lave and Wegner's focus is on situated activities rather than simply the transference of factual knowledge. Participation not only influences the learner, but also the social practice in which the learner and experts are engaged. In keeping with the view taken by $[3,8]$, we propose that the doctoral process is a good example of a cognitive apprenticeship model in operation, where the student learns from expert supervisors, research staff and peers, gradually moving to "full participation" within the community.

\section{Supporting Research}

The idea that creative use of technology could help with the research process has been around for many years, perhaps most famously, in Vannevar Bush's vision of the Memex [5]. The work of Holz et al. [7], on describing what types of support should be provided for Computer Science doctoral students, extends Bloom's taxonomy [2] to describe the core generic skills involved within any Computer Science research project. They categorise research skills as ranging across a number of areas which they label: Organisational, Expressive, Cognitive and MetaCognitive. As the research student masters these skills (and their core subject domain) they move towards becoming full members of their community of practice [9]. While Holz et al. address CS research in particular, and this is also the main focus of this research, we propose that the skills are generic and apply across other research domains. The core skills identified by Holz et al. are informing our design of a toolkit which aims to scaffold a cognitive apprenticeship model, as applied to doctoral education. While individual tools exist to support many of the areas listed above (organisation and expression in particular) they are for the most part not tightly integrated and are usually not explicitly designed to support research. The net result is that research students use of a number of tools that do not provide tight integration and easy migration of data. This paper focuses on a single tool, to support academic writing, which is part of a larger toolkit that 
will assist doctoral students in their studies.

\section{Academic Writing}

Aitchison and Lee highlight that academic research writing is a crucially important, yet inadequately supported process, in doctoral education [1]. They argue that writing skills are best acquired through engagement with a community of practice and through apprenticeship; this suggests the need for collaborative support. They also suggest that writing is a "knowledge-creating" rather than merely knowledge-recording activity. This is supported by other work that describes writing as creative design [11].

Two tools that explore writing from a more creative perspective than that of traditional linear word processors are discussed below.

\section{Writer's Assistant}

The Writer's Assistant focuses on activities that span the entire authoring process, rather than just basic writing functionality [12]. Sharples et al. discuss writing tasks such as brainstorming, note creation, note organising and drafting. They suggest that each task may be represented using specific views, such as networked-notes, linear and structured views. The networked-notes view allows the writer to express ideas as notes and place them into some associative network, similar to existing concept mapping tools. The linear view allows the writer to view the text from beginning to end and perform standard editing activities. Finally the structured view allows the writer to create and alter the structure of the linear text. The tool also provides a number of other options such as templates of standard document types, swapping and merging paragraphs.

\section{iWeaver}

iWeaver [13] builds on the work of Writers Assistant. Just as in the Writer's Assistant the tool provides multiple representations of the document. The representations differ from the Writer's Assistant in that it uses a map view over a network view for their ContextMap view and they argue that the map view is easier to transform into linear text and requires fewer graphical objects to describe relations, proximity and location rather than the arrows and line of a network. The OutlineTree and DocumentView are similar to the structured and linear views in the Writer's Assistant.

\section{Comparisons with Existing Tools}

The most commonly used word processors today typically offer a linear view of documents and are not explicitly designed to support the free association of ideas, thus promoting thinking in a linear manner. Third party concept mapping tools facilitate a freer association of ideas, but the majority were not explicitly designed to support the writing process nor do they integrate multiple representations of the document as does Writer's Assitant and iWeaver. There are a number of online options emerging that allow for collaboration such as Google Docs or Zoho, but these again are limited by a linear focus.

\section{DESIGN METHODOLOGY Participatory Action Research}

This research is using a Participatory Action Research (PAR) methodology [15] and the lead researcher and designer is himself a doctoral candidate who is using the tools as they are being developed ${ }^{1}$. The PAR methodology allows for the involvement of other members of the wider research community within our institution, and outside, becoming participants in the research. The action research aspect suggests we are not only looking to observe practice within the community but that we also want to influence the practices, as mentioned earlier on the work in situated learning [9].

A Participatory Design (PD) methodology [10] complements the PAR approach just mentioned. It is argued that participatory design embodies elements of cognitive apprenticeship [6] where the designer is learning from the practitioner and vice versa. This approach is appropriate in this case as the lead researcher is both a designer and user of the system; this places the researcher in a privileged position with knowledge from both a practice and design perspective. While this position provides an advantage, it may also bias the design if others from the wider community are not involved. Following the PD approach users from the wider community are encouraged to participate in the design process. Muller suggests that the environment in which the users are engaged in the design process is important [10]. He makes the distinction between the design environment and the work context. Both of these environments play a role in this study. Observations and usability testing of users in their usual work context and in a design environment guide the development of the tool. Muller also discusses the idea of a "third hybrid space" that brings the users and the designer together outside their usual environment.

\section{Future Technology Workshop}

A key activity in our research is the holding of "Future Technology Workshops" (FTW) [14] where potential end-users envision future technologies to help support their current activities. A number of workshops are being run with doctoral students and where possible their supervisors and post-doctoral researchers. Workshops are conducted in a "third hybrid space" that lies outside the usual work context of the participants. The particular location we use is a

\footnotetext{
${ }^{1}$ The tool was used in writing this paper
} 
suite of rooms purposely designed to encourage creative group work. A set of requirements for future technology is emerging from these workshops and is informing the design of the overall toolkit. This engagement with the wider community is helping to establish a cohort of active participants in the design process.

\section{DESIGN}

The novel affordance of our approach is that it combines the advantages of the Writer's Assistant and iWeaver approaches and places them in an online environment. This allows tight and loose integration with existing Web 2.0 tools and should allow for a more collaborative, flexible and mobile deployment within a cognitive apprenticeship context.

\section{Prototype Writing Tool: CAWriter}

As the overall objective is to create a CSCW/CSCL toolkit to scaffold the doctoral process, a versatile and flexible development platform needs to be chosen. Using open and flexible Web technologies such as SQL, PHP, JavaScript and Ajax allows for the most flexibility when adding functionality in the future. The toolkit will then inherently be networked allowing for collaboration, integration, the use of Web 2.0 components and the development of widget modules, with relative ease. The Web based option will also guarantee a certain level of cross platform compatibility. It will also provide a level of mobility and the possibility to use multiple devices to interact with the tools, moving towards a ubiquitous computing perspective.
The literature on writing calls for the support and visualisations of a number of tasks such as organising ideas and notes, brainstorming, outlining and draft editing $[12,13]$. We have chosen to implement support for a number of the tasks highlighted in the work of Sharples et al. [12], namely views to support brainstorming, note taking, organising notes, linear planning, outlining and drafting of the text. Following a participatory design approach, these tasks also emerge from observations of research students' and supervisors' current practices.

The design process was divided up into three distinct phases. The basic functionality to support the mapping of notes was implemented in phase one. Phase two implemented the multiple representations of the document. These two phases were followed by a heuristic evaluation. Phase three, still ongoing, involved the integration of collaboration facilities to support cognitive apprenticeship, these have yet to be evaluated.

In the initial implementation the following features are supported: higher order cognitive support via structured and unstructured views of notes and document content. This includes the ability to manipulate both the content and location/size of the note and document objects. It also calls for functionality to distinguish between non-linear and linear ideas, support the co-creation of note maps and the sharing of resources within these views. The following sections will expand upon how these aspects are supported.

Phase One

The Map View (see A in Figure 1) supports brainstorming, note taking and organisation. Objects are created by simply clicking on the background or through an option menu at the bottom left. The

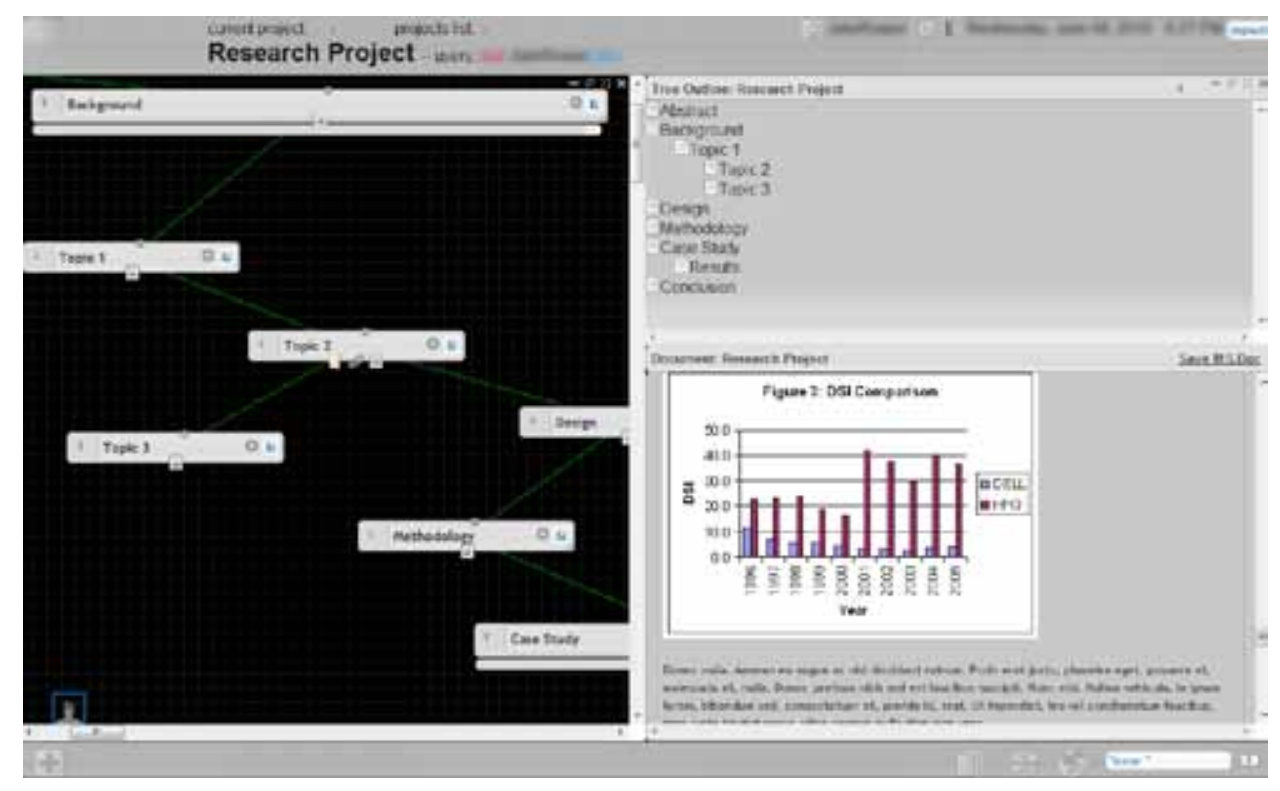

Figure 1. Prototypes multiple views

(A) Map view (B) Tree view (C) Document view 
objects may be moved around the space and may be embedded within other objects. Notes are simply attached to the objects. We chose the iWeaver [13] approach of a "Map View" over the Writer's Assistant's "Network View" to allow users to organise their notes visually rather than in a strict hierarchal fashion. In the Map View the structure is user specified using proximity, positioning and by embedding objects. The Tree and Document Views described below allow for the creation of hierarchies for linear representation and draft edits of documents.

\section{Phase Two}

The Tree View (see B in Figure 1) supports linear planning and outlining and is achieved by adding objects to the tree hierarchy via a simple button. Once objects appear in the Tree View and can be dragged and dropped to where they are desired. The Document View (see C in Figure 1) adds support to the outlining and drafting tasks by providing an output of the document as determined by in the tree structure. This view is similar to the outline view that exists in Microsoft Word, but is not currently available in the online editors such as Google or Zoho Docs. A windows management tool was created to split the screen between the Document/Tree views and the Map View, with the option of making either fullscreen. This ability to economise on screen space helps tackle some of the issues encountered in the iWeaver [13] application where they suggest using the multiple screens.

Additional facilities allow the user to export the document structure to Google Docs, as a MS Word document or as plain HTML.

\section{Phase Three}

This phase is under development at the time of writing. Currently only basic functionality has been implemented that allows multiple users to view and edit content, synchronous/ asynchronous chat and awareness icons that show others users location in the map view as per the CSCW literature. These aspects have yet to be tested extensively to observe how they perform within a cognitive apprentice context, with particular emphasis on $\mathrm{PhD}$ supervision sessions and paper writing.

\section{INITIAL TESTING}

In line with the participatory nature of this work and the preliminary nature of the prototypes development, a number of users close to and involved with the development team were engaged in some early usability testing. In line with both the practicalities of limited resources and the participatory design method, a participatory heuristics approach for evaluation [11] found a number of usability issues in both phases one and two. These included problems with scrolling, lacking the ability to highlight notes, cumbersome resizing of notes, navigating a crowded map-view and slow refresh times. These issues were addressed before phase three was begun.

The underlying framework of Ajax and PHP needs revision in order to speed up the response time and fluidity of the tool.

\section{LIMITATIONS AND FUTURE WORK}

Currently the collaborative features are limited and untested, although collaboration can still occur face-to-face using the tool and users can co-create Map Views remotely. Beyond the basic issues encountered from the participatory heuristic tests, the need for templates of basic document types that the user can call upon to help get them started was suggested. Currently when they log into the system they are presented with a blank space. This is not be the best approach to encourage people to write and does not provide them with as much cognitive support as it could. Access to revisions could also be streamlined. At present there is no way to import documents from other sources such as Microsoft Word other than to copy and paste, although currently not a major issue it will have consequences when collaborating in the future. Finally there is no offline mode, meaning the program is only accessible when the user is connected to the internet. This issue may be resolved using the latest resources provided by HTML 5 where the latest browsers contain databases that allow the application to store data offline.

Future work will initially look at addressing the limitations mentioned above. This means the addition of extra functionality and extensive usability testing. With the introduction of the collaboration features the cognitive apprenticeship and community of practice aspects will come to the fore and be explored in more depth. This will focus on supervisory sessions and writing practices. Following the Meta-Cognitive skills as discussed by Holz et al. [7], utilities will be developed to support such strategies as reflection, self-regulation and monitoring. To this end it is not sufficient to provide utilities to comment or suggest changes to the document but to make explicit the strategies used by experienced academic writers so that the novice can assimilate these practices as they engage in "legitimate peripheral participation" [9].

\section{ACKNOWLEDGEMENTS}

The authors would like to thank the members of the Centre for Research in IT in Education, Trinity College Dublin for their support during this work. 


\section{REFERENCES}

[1] Aitchison, C. and Lee, A. 2006. Research writing: Problems and pedagogies. Teach High Educ, 11, 3, 265-278.

[2] Bloom, B. 1959. Taxonomy of educational objectives. Longmans, New York.

[3] Boud, D. and Lee, A. 2005. Peer learning as pedagogic discourse for research education. Stud High Educ, 30, 5, 501-516.

[4] Brown, J. S., Collins, A. and Duguid, P. 1989. Situated cognition and the culture of learning in Educational researcher, 18, 1

[5] Bush, V. 1979. As we may think. ACM SIGPC Notes, 1,4

Farooq, U., Merkel, C. B., Nash, H., Rosson, M. B., Carroll, J. and Xiao, L. 2005. Participatory Design as Apprenticeship: Sustainable Watershed Management as a Community Computing Application. In Proceedings of the Proceedings of the Proceedings of the 38th Annual Hawaii International Conference on System Sciences (Big Island, Hawaii, USA, January 3-6, 2005). IEEE Computer Society Washington, DC, USA

[7] Holz, H. J., Applin, A., Haberman, B., Joyce, D., Purchase, H. and Reed, C. 2006. Research Methods in Computing: What are they, and how should we teach them? In Proceedings of the Working group reports on ITiCSE on Innovation and technology in computer science education (Bologna, Italy2006). ACM.

[8] Hopwood, N. and McAlpine, L. 2007. Exploring a theoretical framework for understanding doctoral education. In Proceedings of the Annual Conference of the Higher Education Research and Development Society of Australia (Adelaide, July 8-11)

[9] Lave, J. and Wenger, E. 1991.Situated learning: Legitimate peripheral participation. Cambridge Univ Pr.

[10] Muller, M. J. 2002. Participatory design: the third space in $\mathrm{HCl}$. Handbook of $\mathrm{HCl}$, Lawrence Erlbaum, Hillsdale, NJ

[11] Muller, M.J. , Matheson L., Page C. and R. Gallup 1998 Methods \& tools: participatory heuristic evaluation in Interactions, ACM, 5,5

[12] Sharples, M. 1999. How we write: writing as creative design. Routledge, London.

[13] Sharples, M. and Pemberton, L. 1989. Developing a writer's assistant. In Computers and Writing: models and tools, Intellect Limited, Oxford, 22-37

[14] Shibata, H. and Hori, K. 2008. Cognitive support for the organization of Writing. New Generat Comput, 26, 2, 97-124.

[15] Vavoula, G. N. and Sharples, M. 2007. Future technology workshop: A collaborative method for the design of new learning technologies and activities.

Com S Coll Learn, 2, 4, 393-419.
[16] Whyte, W. F. 1989. Advancing scientific knowledge through participatory action research. Sociol Forum, 4, 3, 367-385 